\title{
COMPARISON OF NUMERICAL METHODS FOR THE PROBLEM ARISING IN THE GYROTRON THEORY
}

\author{
J. CEPĪTIS, H. KALIS and A. REINFELDS
}

Institute of Mathematics Latvian Academy of Sciences and University of Latvia

Akadēmijas laukums 1, LV-1524 Rīga, Latvia

E-mail: janis.cepitis@lu.lv; harijs.kalis@lu.lv; reinf@latnet.lv

Received October 1, 2004; revised February 1, 2005

\begin{abstract}
There are considered some aspects for numerical solving of problem with Robin's boundary conditions arising in the gyrotron theory. The single mode case is carefully investigated. The obtained observations make possible to offer the suitable strategy for the numerical solving of the problem for general system of nonstationary gyrotron oscillations.

Key words: finite-difference schemes, gyrotrons, method of lines, Robin's boundary conditions
\end{abstract}

\section{Introduction and Formulation of the Mathematical Model}

In recent papers $[1,2,3]$ we have presented the experience obtained by numerical solving of partial cases of certain problem for time-dependent gyrotron equations. Now we are considering a more general situation of investigated previously problems. Using the obtained as well as previous results we propose a suitable strategy for solving the problem for full system of nonstationary gyrotron oscillations.

A competition between the amplitudes of nonstationary gyrotron oscillations $f_{s}$ and the transverse momentum of electrons $p$ in different modes $(s=1, \ldots, m)$ is described by the corresponding nonlinear system of complex partial differential equations (see, [6])

$$
\left\{\begin{array}{l}
\frac{\partial p}{\partial x}+i\left(|p|^{2}-1\right) p=i \sum_{s=1}^{m} f_{s} \exp \left(i \Delta_{s} x+\Psi_{s}\right) \\
\frac{\partial^{2} f_{s}}{\partial x^{2}}-i \frac{\partial f_{s}}{\partial t}+\delta_{s} f_{s}=\frac{I_{s}}{4 \pi^{2}} \int_{0}^{2 \pi} \int_{0}^{2 \pi} p \exp \left[-i\left(\Delta_{s} x+\Psi_{s}\right)\right] d \theta_{0} d \varphi .
\end{array}\right.
$$

Here $i$ is the imaginary unit, $x \in[0, L]$ and $t \geq 0$ are the normalized axial and time coordinates, for every number of mode $s$ correspondingly $\Delta_{s}$ is the frequency mis- 
match, $\delta_{s}=\delta_{s}(x)$ describes variation of critical frequencies, $I_{s}$ is the dimensionless current, $\Psi_{s}=a_{s} t+m_{s} \varphi$ is the phase of the mode, $a_{s}, m_{s}$ are the physical constants, $0 \leq \varphi<2 \pi$. The initial conditions for momentum

$$
\left.p\right|_{x=0}=\exp \left(i \theta_{0}\right)
$$

depend on parameter $0 \leq \theta_{0}<2 \pi$.

In order to study properties of numerical methods we investigate the single mode case having also in mind special restrictions on the variable $p$. If

$$
|p|^{2}=1+C, \quad C=\text { const } \leq 0, \quad m=1, \quad f_{1}=f
$$

the nonlinear system of gyrotron equations for determination of function $f(t, x)$ reduces to the linearized complex integro-differential equation:

$$
i \frac{\partial f}{\partial t}=\frac{\partial^{2} f}{\partial x^{2}}+\delta f-i I e^{-i \Delta_{*} x} \int_{0}^{x} f(t, \xi) e^{i \Delta_{*} \xi} d \xi,
$$

where $\Delta_{*}=\Delta+C$. We formulate boundary conditions of Robin's type for the function $f(t, x)$ in the following form

$$
f(t, 0)=0, \quad \frac{\partial f(t, L)}{\partial x}=-i \gamma f(t, L)
$$

with some positive constant $\gamma$. The initial condition is presented in the form

$$
f(0, x)=\phi(x)
$$

where $\phi(x)$ is a given complex function.

Using the notation

$$
y(t, x)=\int_{0}^{x} f(t, \xi) e^{i \Delta_{*} \xi} d \xi
$$

and the transformation $\bar{f}=e^{i \Delta_{*} x} f$, the integro-differential equation (1.1) can be written in the form of two partial differential equations.

$$
\left\{\begin{array}{l}
\frac{\partial \bar{f}}{\partial t}=-i\left(\frac{\partial^{2} \bar{f}}{\partial x^{2}}-2 i \Delta_{*} \frac{\partial \bar{f}}{\partial x}+\left(\delta-\Delta_{*}^{2}\right) \bar{f}\right)-I y(t, x), \\
\frac{\partial y}{\partial x}=\bar{f}(t, x),
\end{array}\right.
$$

and conditions (1.2), (1.3) are formulated as follows

$$
\left\{\begin{array}{l}
\bar{f}(t, 0)=0, \quad \frac{\partial \bar{f}(t, L)}{\partial x}=-i\left(\gamma-\Delta_{*}\right) \bar{f}(t, L), \\
\bar{f}(0, x)=e^{i \Delta_{*} x} \phi(x) .
\end{array}\right.
$$

In section 2 the case $\delta=$ const is considered. We reduce the main problem to the boundary-value problem for the ordinary differential equations considering the quasistationary solutions. In sections 3-5 we apply the method of lines and finitedifference schemes for the more general case. Finally, in section 6 we present results of numerical experiments. The main conclusions of our development are gathered in section 7 . 


\section{The Quasistationary Solution of the Differential Problem}

We represent the quasistationary solution of the problem $(1.4),(1.5)$ in the following form

$$
\bar{f}(t, x)=g(x) \exp (i \alpha t), \quad y(t, x)=w(x) \exp (i \alpha t),
$$

where $\alpha$ is a complex number $\alpha=\alpha_{1}+i \alpha_{2}, \alpha_{2}$ is a temporal damping factor: if $\alpha_{2}>0$, the solution of (1.4) decreases in time, if $\alpha_{2}<0$, the solution increases, and for $\alpha_{2}=0$ the solution is oscillating in time.

Substituting functions (2.1) into equation (1.4) and boundary conditions (1.5), we obtain the boundary-value problem of Sturm-Liouville type for the system of two ordinary differential equations

$$
\left\{\begin{array}{l}
g^{\prime \prime}(x)-2 i \Delta_{*} g^{\prime}(x)+\lambda g(x)-\operatorname{Iiw}(x)=0 \\
w^{\prime}(x)=g(x) \\
g(0)=w(0)=0, \quad g^{\prime}(L)=-i\left(\gamma-\Delta_{*}\right) g(L)
\end{array}\right.
$$

where $\lambda=\alpha+\delta-\Delta_{*}^{2}$ is a complex eigenvalue.

If $\delta=$ const, then applying a standard scheme in order to solve this linear boundary-value problem we obtain a transcendental complex equation for the calculation of eigenvalues

$$
\mu_{3} \kappa_{3}\left(\mu_{2}-\mu_{1}\right)+\mu_{2} \kappa_{2}\left(\mu_{1}-\mu_{3}\right)+\mu_{1} \kappa_{1}\left(\mu_{3}-\mu_{2}\right)=0,
$$

where $\kappa_{j}=\left(\mu_{j}-i\left(\Delta_{*}-\gamma\right)\right) \exp \left(\mu_{j} L\right), \mu_{j}, j=1,2,3$ are the three roots of the following complex cubic equation:

$$
\mu^{3}-2 i \Delta_{*} \mu^{2}+\lambda \mu-I i=0 .
$$

The roots of equation $(2.3) \lambda^{(k)}, k=1,2, \ldots$ are sorted in increasing order of their real parts.

The corresponding complex eigenfunctions $g^{(k)}(x), w^{(k)}(x)$ are obtained from problem (2.2) for $\lambda=\lambda^{(k)}$ and the quasistationary solution of the problem (1.4), (1.5) for every eigenvalue $\alpha^{(k)}=\lambda^{(k)}-\delta+\Delta_{*}^{2}$ is given in the form

$$
f^{(k)}(t, x)=\exp \left(-i \Delta_{*} x\right) g^{(k)}(x) \exp \left(i \alpha^{(k)} t\right) .
$$

Particularly, if $\alpha_{2}^{(k)}=\operatorname{Im}\left(\lambda^{(k)}\right)>0$ than these solutions asymptotically tend to zero, if $t$ tends to infinity.

\section{Discrete Grids and Approximation of Derivatives}

For approximation of derivatives we consider two types of discrete grids.

1) A uniform grid is defined by grid points

$$
x_{j}=j h, \quad j=0, \ldots, n, \quad h=\frac{L}{n},
$$


where $h$ is the space step.

2) A nonuniform grid, grid points of which are the roots of the Chebyshev polynomials of the second kind [2]

$$
x_{j}=0.5 L(1-\cos (\pi j / n)), \quad j=0, \ldots, n .
$$

Using grid points (3.1) or (3.2) we can approximate partial derivatives $\frac{\partial}{\partial x}, \frac{\partial^{2}}{\partial x^{2}}$ in equations (1.4) for a fixed time moment $t$ with matrixes $D, D^{2}$ of derivatives in the form

$$
f_{h}^{\prime}=D f_{h}, \quad f_{h}^{\prime \prime}=D^{2} f_{h}, \quad y_{h}^{\prime}=D y_{h}, \quad \bar{f}_{h}^{\prime}=D \bar{f}_{h}, \quad \bar{f}_{h}^{\prime \prime}=D^{2} \bar{f}_{h},
$$

where

$$
\begin{array}{ll}
f_{h}=\left(f_{0}, f_{1}, \ldots, f_{n}\right), & f_{h}^{\prime}=\left(f_{0}^{\prime}, f_{1}^{\prime}, \ldots, f_{n}^{\prime}\right), \quad f_{h}^{\prime \prime}=\left(f_{0}^{\prime \prime}, f_{1}^{\prime \prime}, \ldots, f_{n}^{\prime \prime}\right), \\
y_{h}=\left(y_{0}, y_{1}, \ldots, y_{n}\right), & y_{h}^{\prime}=\left(y_{0}^{\prime}, y_{1}^{\prime}, \ldots, y_{n}^{\prime}\right), \quad x_{h}=\left(x_{0}, x_{1}, \ldots, x_{n}\right),
\end{array}
$$

are the vector-columns of the corresponding values of the grid and grid functions

$$
f_{j}=f\left(t, x_{j}\right), \quad f_{j}^{\prime}=\frac{\partial f\left(t, x_{j}\right)}{\partial x}, f_{j}^{\prime \prime}=\frac{\partial^{2} f\left(t, x_{j}\right)}{\partial x^{2}} .
$$

It follows from the Lagrange interpolation formula (see, [5]), that the elements of matrix $D$ are given in the form

$$
D_{j, k}=\frac{d l_{k}\left(x_{j}\right)}{d x}, \quad j, k=0, \ldots, n,
$$

where

$$
l_{k}(x)=\frac{\omega(x)}{\omega^{\prime}\left(x_{k}\right)\left(x-x_{k}\right)}, \quad \omega=\prod_{k=0}^{n}\left(x-x_{k}\right)
$$

are the elementary Lagrange multipliers.

It must be mentioned that for the nonuniform grid (3.2) if the grid points are chosen as the roots of the Chebyshev polynomials of the second kind the interpolation error is small [5].

Next we define a uniform temporal grid:

$$
t_{m}=m \tau, \quad m=1,2, \ldots, M,
$$

where a corresponding time-step $\tau$ is used. We substitute the continuous function $f$ on this grid by the discrete values $f_{j}^{m} \approx f\left(t_{m}, x_{j}\right)$. The corresponding derivatives are approximated by finite-differences

$$
\begin{aligned}
& \frac{\partial^{2} f\left(t_{m}, x_{j}\right)}{\partial x^{2}} \approx \frac{f_{j+1}^{m}-2 f_{j}^{m}+f_{j-1}^{m}}{h^{2}}, \quad \frac{\partial f\left(t_{m}, x_{j}\right)}{\partial t} \approx \frac{f_{j}^{m+1}-f_{j}^{m}}{\tau}, \\
& \frac{\partial f\left(t_{m}, x_{j}\right)}{\partial x} \approx \frac{f_{j+1}^{m}-f_{j-1}^{m}}{2 h}, \quad \frac{\partial f\left(t_{m}, L\right)}{\partial x} \approx \frac{f_{n}^{m}-f_{n-1}^{m}}{h}, \quad j=1, \ldots, n-1 .
\end{aligned}
$$




\section{Method of Lines for Solving Differential Problems}

Considering only the spatial discretization of problem (1.4), (1.5), when the variable $x$ is discretized and the variable $t$ is continuous, and using the matrixes $D, D^{2}$ of derivatives in the grids (3.1) or (3.2) we obtain for grid functions $f_{h}=f_{h}(t), y_{h}=$ $y_{h}(t)$ the system of $(n-1)$ complex ordinary differential equations (the boundary conditions (1.2) are taken into account during construction of matrixes $D, D^{2}$ )

$$
\frac{d f_{h}}{d t}=B f_{h}-I E^{-} y_{h}, \quad D y_{h}=E^{+} f_{h}
$$

where $B=-i\left(D^{2}+\delta E\right), E^{ \pm}=\operatorname{diag}\left(\exp \left( \pm i \Delta_{*} x_{h}\right)\right)$ are quadratic $(n-1) \times(n-1)$ matrixes, $E^{ \pm}, E$ are the corresponding diagonal matrixes and the unit matrix. The initial conditions are defined" in the form

$$
f_{h}(0)=\phi_{h},
$$

where

$$
\phi_{h}=\left(\phi_{1}, \ldots, \phi_{n-1}\right), \quad \phi_{j}=\phi\left(x_{j}\right), \quad j=1, \ldots, n-1 .
$$

Excluding the vector $y_{h}$ in (4.1) we derive the initial-value problem:

$$
\frac{d f_{h}}{d t}=G f_{h}, \quad f_{h}(0)=\phi_{h},
$$

where $G=B-I E^{-}\left(D^{-1} E^{+}\right)$.

The solution $f_{h}(t)$ of the problem (4.2) can be obtained in two forms:

1) by using matrix-exponent function

$$
f_{h}(t)=\exp (G t) \phi_{h},
$$

2) by using the spectral decomposition of matrix $G=R D_{0} R^{-1}$, where $D_{0}$ is the diagonal matrix of eigenvalues, $R$ is the eigenvectors matrix with corresponding eigenvectors as columns of the matrix

$$
f_{h}(t)=R \exp \left(D_{0} t\right)\left(R^{-1} \phi_{h}\right) .
$$

Using matrixes $D, D^{2}$ and the approximation of integrals $y\left(t, x_{j}\right)$ we obtain for solving the integro-differential equation (1.1) the system (4.2), where the elements of matrix $G$ are given in the following form

$$
\left\{\begin{aligned}
G_{k, j}=B_{k, j} & -I \exp \left(-i \Delta_{*} x_{k}\right)\left(h_{j} B_{j} \exp \left(i \Delta_{*} x_{j-1}\right)\right. \\
& \left.+h_{j+1} A_{j} \exp \left(i \Delta_{*} x_{j}\right)\right), \quad j=1, \ldots, k-1, \\
G_{k, k}=B_{k, k} & -I h_{k} \exp \left(-i \Delta_{*} h_{k}\right) B_{k},
\end{aligned}\right.
$$

where $B_{k, j}$ are elements of matrix $B$;

$$
\begin{gathered}
B_{j}=\exp \left(i \Delta_{*} h_{j}\right)\left(\left(\Delta_{*} h_{j}\right)^{-2}+\left(i \Delta_{*} h_{j}\right)^{-1}\right)-\left(\Delta_{*} h_{j}\right)^{-2}, \\
A_{j}=\left(\Delta_{*} h_{j}\right)^{-2}-\left(i \Delta_{*} h_{j}\right)^{-1}-\exp \left(i \Delta_{*} h_{j}\right)\left(\Delta_{*} h_{j}\right)^{-2}
\end{gathered}
$$

are coefficients of the quadrature formula, $h_{j}=x_{j}-x_{j-1}, k=1, \ldots, n$. 


\section{Discrete Approximations in Time and Finite-Difference Schemes}

Using the uniform time-grid $t_{m}=m \tau, m=0,1,2, \ldots, M$ we can consider following approximation of the system (4.1):

$$
\left\{\begin{array}{l}
\frac{f_{h}^{m+1}-f_{h}^{m}}{\tau}=B\left(\sigma f_{h}^{m+1}+(1-\sigma) f_{h}^{m}\right)-I E^{-}\left(\sigma y_{h}^{m+1}+(1-\sigma) y_{h}^{m}\right), \\
D y_{h}^{m}=E^{+} f_{h}^{m}, \quad f_{h}^{0}=\phi_{h},
\end{array}\right.
$$

where $\sigma \in[0,1]$ is the weight coefficient of the scheme,

$$
f_{h}^{m}=f_{h}\left(t_{m}\right), \quad m=0,1, \ldots, M
$$

By eliminating vector $y_{h}^{m}$ from the system we obtain the two level finite-difference scheme

$$
f_{h}^{m+1}=\bar{G} f_{h}^{m}
$$

where the transition matrix $\bar{G}$ has the form

$$
\bar{G}=\left(\tau^{-1} E-\sigma\left(B-I E^{-} D^{-1} E^{+}\right)\right)^{-1}\left(\tau^{-1} E+(1-\sigma)\left(B-I E^{-} D^{-1} E^{+}\right)\right) .
$$

It follows from (5.1) that $f_{h}^{m}=(\bar{G})^{m} \phi_{h}$.

The discrete approximation in time for the integro-differential equations (1.1) is given by

$$
\frac{f_{h}^{m+1}-f_{h}^{m}}{\tau}=G\left(\sigma f_{h}^{m+1}+(1-\sigma) f_{h}^{m}\right),
$$

where $f_{h}^{0}=\phi_{h}, m=0,1, \ldots, M$ and the elements of matrix $G$ are written in the form (4.4), $\sigma \in[0,1]$.

In this case we can rewrite (5.2) in the form (5.1), where

$$
\bar{G}=\left(\tau^{-1} E-\sigma G\right)^{-1}\left(\tau^{-1} E+(1-\sigma) G\right) .
$$

For the finite-difference approximation of integro-differential equation (1.1) using the uniform grid (3.1) we apply the method of lines in the form (4.2) with matrix $G$ written in three-diagonal and left-triangle forms. The nonzero elements of this matrix are defined as

$$
\left\{\begin{array}{c}
G_{k, k}=i\left(2 h^{-2}-\delta\right)-I h \exp \left(-i \Delta_{*} h\right) B_{1}, G_{k, k+1}=-i h^{-2} \\
G_{k, j}=-\left(i h^{-2} \delta_{j, k-1}+I h\left(B_{1} \exp \left(-i \Delta_{*}(k-j+1) h\right)\right.\right. \\
\left.\left.\quad+A_{1} \exp \left(-i \Delta_{*}(k-j) h\right)\right)\right)
\end{array}\right.
$$

where $\delta_{j, k-1}$ is the Kronecker delta function, $A_{1}, B_{1}$ are coefficients of the quadrature formula with $h_{j}=h, j=1, \ldots, k-1, k=1, \ldots, n-1$.

Boundary conditions (1.2) are approximated in the form 


$$
\frac{f_{n}-f_{n-1}}{h}=-i \gamma f_{n}
$$

thus the element $G_{n-1, n-1}$ of matrix $G$ must be supplemented with additional term $-i h^{-2} /(1+i h \gamma)$.

For finite-difference approximations in space coordinate by using the uniform grid (3.1) and time we can consider a two level finite-difference scheme (5.2), where elements of matrix $G$ are given in the form (5.4). The transition matrix $\bar{G}$ is defined by (5.3).

In the case of $I=0$ the stability condition of this scheme is $\sigma \geq 0.5$ (see, [3]). This condition is valid for $I \neq 0$.

A similar finite-difference scheme for solving differential equations (1.1) can be obtained from (4.1), where matrixes $D, B$ are defined using the finite differences in space. Difference equations approximate the differential equation with the second order both in space and time, if $\sigma=1 / 2$, and with the first order in time, if $\sigma \neq 1 / 2$ (see, [4]). Boundary conditions (1.2) are approximated only with the first order in space. Therefore, problem (1.1) - (1.3) is approximated only with the first order of accuracy.

\section{Numerical Experiments with Different Methods}

All numerical results are obtained by means of computer programs MATLAB and MAPLE for the following parameters:

$$
\begin{aligned}
& \gamma=2, \quad L=15, \quad \delta=0, \quad \delta=\delta(x)=\frac{1}{900} \sinh (x-7.5), \quad C=0, \\
& I=0, \quad I=0.01, \quad \Delta= \pm 10, \quad \phi=\sin (\pi x / L) .
\end{aligned}
$$

The first 15 eigenvalues $\bar{\lambda}^{(k)}, \quad k=1, \ldots, 15$ of matrix $G(n=25)$ in (4.2) are presented in Tab. $1(\Delta=10)$.

For the nonuniform grid (3.2) with $n=25, \delta=0$ the first 15 eigenvalues $\bar{\lambda}^{(k)}$ of matrix $G$ of the differential equations (4.2) are equal to $i\left(\lambda^{(k)}+\Delta_{*}^{2}\right)$ and numerical approximations have the accuracy of 4 decimal digits, where $\lambda^{(k)}$ are the roots of equation (2.3). The maximal value $M_{1}=\operatorname{Im}\left(\bar{\lambda}^{(n)}\right)$ of eigenvalues of matrix $G$ increase, but $M_{0}=-\operatorname{Re}\left(\bar{\lambda}^{(n)}\right)$ decrease if the number of grid points $n$ is enlarged. As an example, if

$$
I=1, \quad \Delta_{*}=2, \quad \delta=0,
$$

then for the nonuniform grid (3.2)

$$
\begin{aligned}
& M_{1}=331.13, \quad M_{0}=0.050, \quad(n=25), \\
& M_{1}=5271.6, \quad M_{0}=0.0001, \quad(n=50) .
\end{aligned}
$$

These values depend little on $\Delta_{*}$ and $I$. Therefore, function $\exp \left(\left(-M_{0}+i M_{1}\right) t\right)$ slowly decreases and rapidly oscillates in time. The calculations show that

$$
\operatorname{Re}\left(\bar{\lambda}^{(k)}\right)<0, \quad k=1, \ldots, n
$$


Table 1. The first 15 eigenvalues $\bar{\lambda}^{(k)}$ for matrix $G$.

\begin{tabular}{lllll}
\hline$k$ & $\delta=I=0$ & $\delta(x), I=0$ & $\delta=0, I=0.01$ & $\delta(x), I=0.01$ \\
\hline 1 & $-.0029+.0437 \mathrm{i}$ & $-.0147-.0387 \mathrm{i}$ & $-.0039+.0336 \mathrm{i}$ & $-.0145-.0287 \mathrm{i}$ \\
2 & $-.0118+.1748 \mathrm{i}$ & $-.0350-.1678 \mathrm{i}$ & $-.0129+.1689 \mathrm{i}$ & $-.0364-.1615 \mathrm{i}$ \\
3 & $-.0272+.3933 \mathrm{i}$ & $-.0565+.3912 \mathrm{i}$ & $-.0305+.3856 \mathrm{i}$ & $-.0597-.3832 \mathrm{i}$ \\
4 & $-.0497+.6989 \mathrm{i}$ & $-.0853+.7024 \mathrm{i}$ & $-.0556+.6926 \mathrm{i}$ & $-.0916+.6957 \mathrm{i}$ \\
5 & $-.0808+1.091 \mathrm{i}$ & $-.1249+1.099 \mathrm{i}$ & $-.0898+1.088 \mathrm{i}$ & $-.1343+1.095 \mathrm{i}$ \\
6 & $-.1230+1.570 \mathrm{i}$ & $-.1809+1.580 \mathrm{i}$ & $-.1310+1.571 \mathrm{i}$ & $-.1891+1.581 \mathrm{i}$ \\
7 & $-.1807+2.133 \mathrm{i}$ & $-.2664+2.140 \mathrm{i}$ & $-.1849+2.137 \mathrm{i}$ & $-.2707+2.145 \mathrm{i}$ \\
8 & $-.2636+2.773 \mathrm{i}$ & $-.4141+2.736 \mathrm{i}$ & $-.2632+2.776 \mathrm{i}$ & $-.4141+2.740 \mathrm{i}$ \\
9 & $-.3863+3.458 \mathrm{i}$ & $-.4775+3.253 \mathrm{i}$ & $-.3847+3.457 \mathrm{i}$ & $-.4754+3.252 \mathrm{i}$ \\
10 & $-.4749+4.116 \mathrm{i}$ & $-.3912+3.970 \mathrm{i}$ & $-.4779+4.112 \mathrm{i}$ & $-.3935+3.966 \mathrm{i}$ \\
11 & $-.4268+4.886 \mathrm{i}$ & $-.3487+4.839 \mathrm{i}$ & $-.4324+4.888 \mathrm{i}$ & $-.3547+4.840 \mathrm{i}$ \\
12 & $-.3780+5.822 \mathrm{i}$ & $-.3266+5.802 \mathrm{i}$ & $-.3802+5.826 \mathrm{i}$ & $-.3289+5.806 \mathrm{i}$ \\
13 & $-.3497+6.866 \mathrm{i}$ & $-.3132+6.854 \mathrm{i}$ & $-.3487+6.866 \mathrm{i}$ & $-.3122+6.855 \mathrm{i}$ \\
14 & $-.3319+8.002 \mathrm{i}$ & $-.3041+7.994 \mathrm{i}$ & $-.3339+8.000 \mathrm{i}$ & $-.3060+7.992 \mathrm{i}$ \\
15 & $-.3197+9.228 \mathrm{i}$ & $-.2976+9.223 \mathrm{i}$ & $-.3230+9.229 \mathrm{i}$ & $-.3009+9.224 \mathrm{i}$ \\
\hline
\end{tabular}

for all parameters and methods. At first the values $\left|\operatorname{Re}\left(\bar{\lambda}^{(k)}\right)\right|$ increase, if $k$ enlarged, then those reach the maximum and then asymptotically tend to zero.

The algorithm of method of lines can be improved, if the last eigenvalues with large $M_{1}$ are eliminated. If $n=100, \delta=0$, then the first $n_{1}=60$ eigenvalues of matrix $G$ coincide with the roots of equation (2.3), but the last $n_{2}=40$ eigenvalues are not close to the roots of equation (2.3). Fig. 1 ( $I=0.01, \delta=0, \Delta=10)$ shows the first 80 eigenvalues $\alpha^{(k)}=-i \bar{\lambda}^{(k)}, k=1, \ldots, 80$ with 20 "parasitic" eigenvalues.

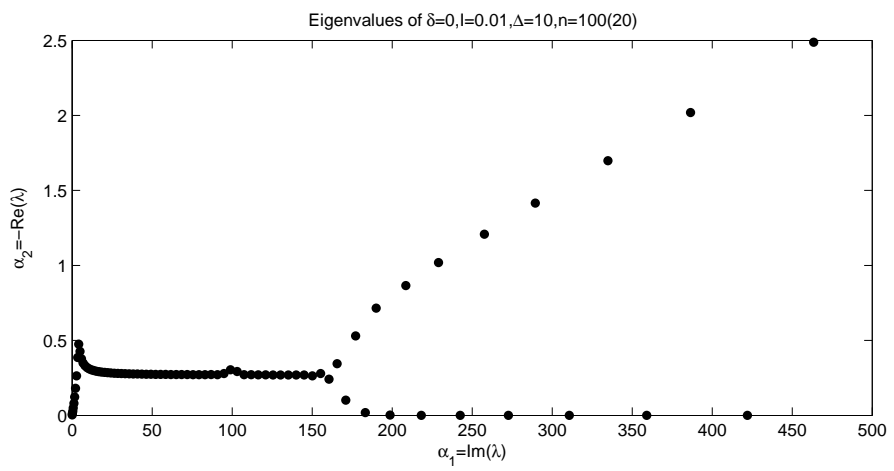

Figure 1. Eigenvalues $\alpha^{(k)}$ of $\delta=0, I=0.01, \Delta=10, n=100(20)$.

Therefore, we can use the spectral decomposition method (4.3) for solving the system of ODEs (4.2)

$$
f_{h}(t)=R_{*} \exp \left(D_{0}^{*} t\right)\left(R^{-1} \phi_{h}\right)_{*},
$$


or

$$
f_{h}(t)=R_{*} \exp \left(D_{0}^{*} t\right)\left(R_{*}^{\prime} R_{*}\right)^{-1} R_{*}^{\prime} \phi_{h},
$$

where $R_{*}$ is the matrix $R$ without the last $n_{2}$ columns, $R_{*}^{\prime}$ is the transpose matrix of $R_{*}, D_{0}^{*}$ is the quadratic matrix of $D_{0}$ without the last $n_{2}$ columns and rows, and $\left(R^{-1} \phi\right)_{*}$ is the vector-column $\left(R^{-1} \phi\right)$ without the last $n_{2}$ rows. The expressions (6.1), (6.2) are in the form $f_{h}(t)=R_{*} \exp \left(D_{0}^{*} t\right) w_{h}^{*}$, where $w_{h}^{*}$ is the vector-column $w_{h}$ without the last $n_{2}$ rows, but $w_{h}, w_{h}^{*}$ are the following solutions of algebraic systems equations:

1) the system with full matrix $R(\operatorname{order} n)-w_{h}=R^{-1} \phi_{h}$ in (6.1),

2 ) the system with matrix $R_{*}^{\prime} R_{*}\left(\operatorname{order} n_{1}\right)$ from the least-squares approximation or Gauss transformation $-w_{h}^{*}=\left(R_{*}^{\prime} R_{*}\right)^{-1} R_{*}^{\prime} \phi_{h}$ in (6.2).

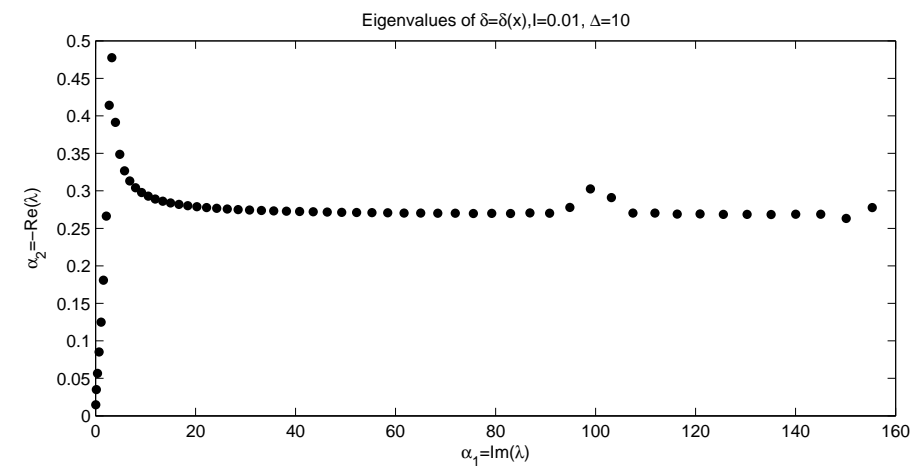

Figure 2. Eigenvalues $\alpha^{(k)}$ of $\delta(x), I=0.01, \Delta=10, n=100(40)$.

Fig. 2, shows the first 60 eigenvalues $\alpha^{(k)}, k=1, \ldots, 60$, where $n=100$; $\delta=\delta(x)$ and $I=0.01, \Delta=10$. Here the values $\left|\operatorname{Re}\left(\bar{\lambda}^{(k)}\right)\right|$ tend to value 0.270 , if the number $k$ is enlarged.

For the uniform grid (3.1) using the finite-difference schemes gives us a possibility to compute accurately only the first 3-5 eigenvalues, if $n \geq 100$ (see Fig. 3 with $n=100, I=0.01, \delta=0, \Delta=10$, here the exact value of $\alpha_{2}$ tends asymptotically to 0.2675 when $\alpha_{1}$ tends to infinity, but the corresponding value obtained by the finite-difference method tends to zero).

Next we present results of numerical experiments, when problem $(1.1)-(1.3)$ is solved with different methods.

Results of application of the method of lines $\left(n=100, n_{1}=60\right)$ for values $|f(t, L)|, t \in[0,400]$ are presented in Fig. $4(\delta=0, I=0.01, \Delta=10)$ and Fig. 5 ( $\delta=\delta(x), I=0.01, \Delta=10$ ). If $\delta=0$ then the solution tends to zero very slowly.

Tab. 2 shows that the calculation with $n_{2}=0.4 n$, using expressions (6.1), (6.2) are more stable in comparison with $n_{2}=0$ from (6.1). 


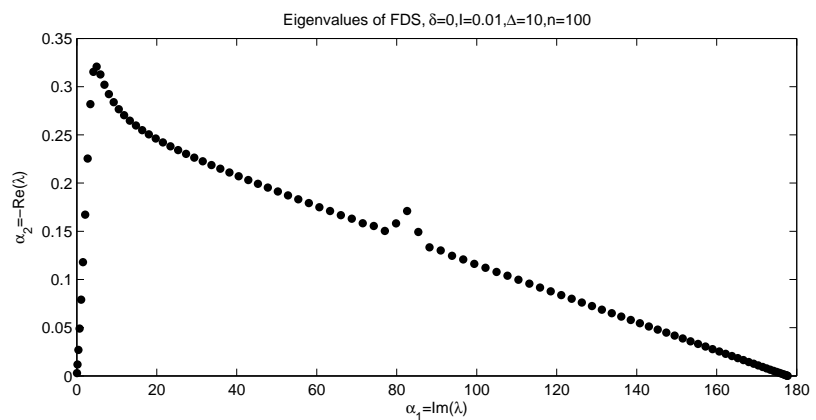

Figure 3. Eigenvalues $\alpha^{(k)}$ of $\delta=0, I=0.01, \Delta=10, n=100$ for finite-difference scheme.

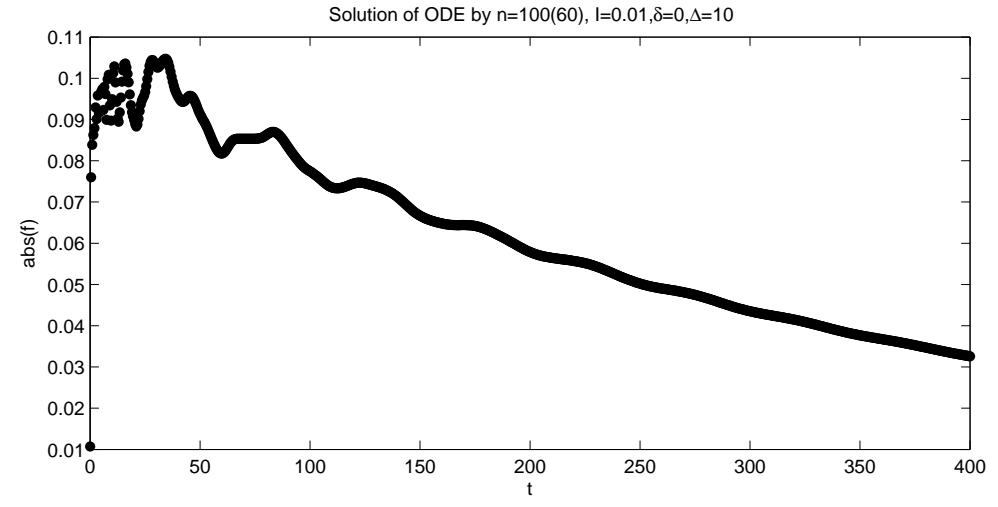

Figure 4. Solution $|f(t, 15)|$ by $n=100, I=0.01, \delta=0, \Delta=10$.

Table 2. The dependence of value $F_{n}=|f(1, L)|$ from $n$ for $I=0.01, \delta=\delta(x), \Delta=10$.

\begin{tabular}{rccc}
\hline$n$ & $(6.1), n_{2}=0.4 n$ & $(6.2), n_{2}=0.4 n$ & $(6.1), n_{2}=0$ \\
\hline 5 & 0.0809 & 0.0842 & 0.0776 \\
10 & 0.0918 & 0.0990 & 0.1008 \\
20 & 0.0983 & 0.1018 & 0.1049 \\
40 & 0.1001 & 0.1003 & 0.1003 \\
60 & 0.1001 & 0.1002 & 0.0996 \\
80 & 0.1003 & 0.1003 & 0.1003 \\
100 & 0.1003 & 0.1003 & 0.1011 \\
\hline
\end{tabular}

\section{Conclusions}

It follows from the numerical experiments that for the two level approximation the time step $\tau$ must be small. For the finite-difference scheme the number of grid points is increased $(n \geq 200)$. Therefore this requires large computer time and it is more suitable to use the nonuniform grid and the algorithm based on the method of lines. 


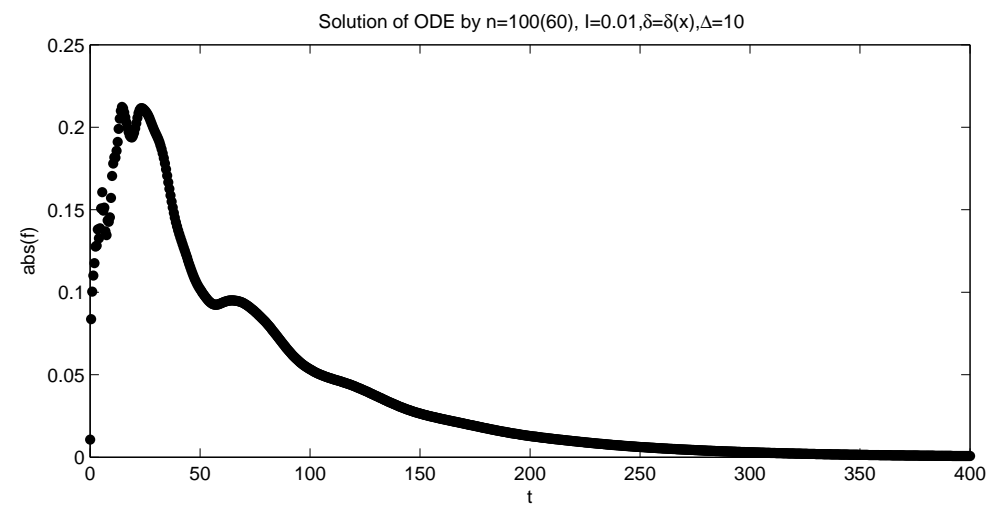

Figure 5. Solution $|f(t, 15)|$ by $n=100, I=0.01, \delta=\delta(x), \Delta=10$.

The finite-difference schemes (5.2), (5.4) are stable for $\sigma \geq 0.5$, but they are not sufficiently accurate. Using the matrix of derivatives on the uniform grid it is possible to make calculations only for small $n(n \leq 25)$, otherwise the calculations are unstable.

\section{References}

[1] M.I. Airila, O. Dumbrajs, A. Reinfelds and U. Strautiņš. Nonstationary oscillations in gyrotrons. Phys. Plasmas, 8(10), 4608 - 4612, 2001.

[2] T. Cirulis, H. Kalis and O. Lietuvietis. Comparative analysis by means of finite differences and DM methods for linearized problem of gyrotrons. Mathematical modelling and analysis, 9(2), 127 - 136, 2004.

[3] O. Dumbrajs, H. Kalis and A. Reinfelds. Numerical solution of single-mode gyrotron equation. Mathematical modelling and analysis, 9(1), 25 - 38, 2004.

[4] A.A. Samarskii. The theory of difference schemes. Marcel Dekker, New York, NY, 2001.

[5] G.A. Watson. Approximation theory and numerical methods. John Wiley \& Sons, Chichester etc., 1980.

[6] N.A. Zavolsky, G.S. Nusinovich and A.B. Pavelyev. Stability of single-mode oscillations and nonstationary processes in gyrotrons with very large low quality factor resonators. In: Gyrotrons. Academy of Sciences of USSR, Gorky, 84 - 112, 1989. (in Russian) 


\section{Skaitinių metodų girotrono teorijos uždaviniuose palyginimas}

J. Cepītis, H. Kalis, A. Reinfelds

Straipsnyje nagrinėjami girotrono teorijos uždavinių su Robino kraštine sąlyga kai kurie skaitinio sprendimo metodų aspektai. Atidžiai nagrinèjamas vienos modos atvejis ir gauti pastebejjimai leidžia sudaryti tinkamą skaitinio šio uždavinio sprendimo strategiją bendrajai girotrono lygčiu sistemai, aprašančiai jo nestacionarius virpesius. 